\title{
A Determinant Expression for the Generalized Bessel Polynomials
}

\author{
Sheng-liang Yang and Sai-nan Zheng \\ Department of Applied Mathematics, Lanzhou University of Technology, Lanzhou, \\ Gansu 730050, China \\ Correspondence should be addressed to Sheng-liang Yang; slyang@lut.cn
}

Received 5 June 2013; Accepted 25 July 2013

Academic Editor: Carla Roque

Copyright (C) 2013 S.-1. Yang and S.-n. Zheng. This is an open access article distributed under the Creative Commons Attribution License, which permits unrestricted use, distribution, and reproduction in any medium, provided the original work is properly cited.

Using the exponential Riordan arrays, we show that a variation of the generalized Bessel polynomial sequence is of Sheffer type, and we obtain a determinant formula for the generalized Bessel polynomials. As a result, the Bessel polynomial is represented as determinant the entries of which involve Catalan numbers.

\section{Introduction}

The Bessel polynomials form a set of orthogonal polynomials on the unit circle in the complex plane. They are important in certain problems of mathematical physics; for example, they arise in the study of electrical networks and when the wave equation is considered in spherical coordinates. Many other important applications of such polynomials may be found in Grosswald [1, pages 131-149]. The Bessel polynomials [1, 2] are the polynomial solutions of the second-order differential equations

$$
z^{2} y_{n}^{\prime \prime}(z)+(2 z+2) y_{n}^{\prime}(z)=n(n+1) y_{n}(z), \quad y_{n}(0)=1
$$

From this differential equation one can easily derive the formula

$$
y_{n}(z)=\sum_{k=0}^{n}\left(\begin{array}{l}
n \\
k
\end{array}\right)\left(\begin{array}{c}
n+k \\
k
\end{array}\right) k ! \frac{z^{k}}{2^{k}}
$$

Let $a(n, k)$ denote the coefficient of $z^{n-k}$ in $y_{n-1}(z)$. We call $a(n, k)$ a signless Bessel number of the first kind and $b(n, k)=(-1)^{n-k} a(n, k)$ a Bessel number of the first kind. The Bessel number of the second kind $B(n, k)$ is defined to be the number of set partitions of $[n]$ := $\{1,2,3, \ldots, n\}$ into $k$ blocks of size one or two. Han and Seo [3] showed that the two kinds of Bessel numbers are related by inverse formulas, and both Bessel numbers of the first kind and those of the second kind form logconcave sequences. In [4], the Bessel matrices obtained from the Bessel numbers of the first kind $b(n, k)$ and of the second kind $B(n, k)$ are introduced, respectively. It is shown that these matrices can be represented by the exponential Riordan matrices. The relations between the Bessel matrices, Catalan matrices, Stirling matrices, and Fibonacci matrices are also investigated. Recently, [5] showed that Bessel numbers correspond to a special case of the generalized Stirling numbers. Bessel polynomials have the following two determinant representations [6]: 


$$
\begin{aligned}
& 2^{n} y_{n}(z)=\left|\begin{array}{ccccc}
2(1+n z) & -1 & 0 & \cdots & 0 \\
-2 z(2+n z) & 2(1+n z) & -2 & \cdots & 0 \\
2 z^{2}(3+n z) & -2 z(2+n z) & 2(1+n z) & \cdots & 0 \\
\vdots & \vdots & \vdots & \ddots & \vdots \\
2(-z)^{n-1}(n+n z) & 2(-z)^{n-2}(n-1+n z) & 2(-z)^{n-3}(n-2+n z) & \cdots & 2(1+n z)
\end{array}\right| \text {, } \\
& y_{n}(z)=\left|\begin{array}{ccccccc}
(2 n-1) z & -1 & 0 & \cdots & 0 & 0 & 0 \\
1 & (2 n-3) z & -1 & \cdots & 0 & 0 & 0 \\
0 & 1 & (2 n-5) z & \cdots & 0 & 0 & 0 \\
\vdots & \vdots & \vdots & \ddots & \vdots & \vdots & \vdots \\
0 & 0 & 0 & \cdots & 1 & z & -1 \\
0 & 0 & 0 & \cdots & 0 & 1 & 1
\end{array}\right| .
\end{aligned}
$$

In Eğecioğlu [7], a $(n+1) \times(n+1)$ Hankel determinant $\widetilde{H}_{n}(z)$ is introduced by $\widetilde{H}_{n}(z)=\operatorname{det}\left[e_{i+j}(z)\right]_{0 \leq i, j \leq n}$, where $e_{k}(z)=\sum_{m=0}^{k}\left(z^{m} / m !\right)$, and a closed form evaluation of this determinant in terms of the Bessel polynomials is given. Hence another determinant expression for the Bessel polynomials is given by $c_{n} y_{n}(2 z)=H_{n}(z)$ with $H_{n}(z)=$ $\operatorname{det}\left[a_{i+j}(z)\right]_{0 \leq i, j \leq n}$, where $c_{n}=(-1)^{n(n+1) / 2} 2^{n} \prod_{j=1}^{n}\left(j !^{2} /(2 j) !^{2}\right)$ and $a_{k}(z)=\sum_{m=0}^{k}\left(z^{m} /(k-m) !\right)$.

In this paper, using the production matrix of Bessel matrix which is represented as exponential Riordan array, we show that a variation of the generalized Bessel polynomial sequence is of Sheffer type, and we give a determinant formula for the generalized Bessel polynomials. As a result, the Bessel polynomial is represented as determinant the entries of which involve Catalan numbers.

\section{Exponential Riordan Array}

The concept of Riordan array was introduced by Shapiro et al. [8] in 1991; then the concept is generalized to the exponential Riordan array by many authors [4, 9-13]. Let $g(t)=1+\sum_{n=1}^{\infty} g_{n}\left(t^{n} / n !\right), h(t)=\sum_{n=1}^{\infty} h_{n}\left(t^{n} / n !\right)$ with $h_{1} \neq 0$ be two formal power series. An exponential Riordan array is an infinite lower triangular array $A=\left(a_{n, k}\right)_{n, k \geq 0}$ whose $k$ th column has exponential generating function $(1 / k !) g(t) h(t)^{k}$. The array corresponding to the pair $g(t)$, $h(t)$ is denoted by $A=[g(t), h(t)]$. The group law is then given by $[g(t), h(t)][d(t), l(t)]=[g(t) d(h(t)), l(h(t))]$. From the definition it follows at once that the bivariate generating function of $A=[g(t), h(t)]$, namely, $G_{A}(x, t)=$ $\sum_{n, k} a_{n, k} x^{k}\left(t^{n} / n !\right)$, is given by

$$
G_{A}(x, t)=g(t) \exp (x f(t)) .
$$

Each exponential Riordan array $A=[g(t), h(t)]$ is associated a matrix $P$ called its production matrix [12], which is determined by $P=A^{-1} \bar{A}$, where $\bar{A}$ is the matrix $A$ with its top row removed. The production matrix of an exponential Riordan array is characterized by the following lemma [10].
Lemma 1. Let $A=\left(a_{n, k}\right)_{n, k \geq 0}=[g(t), h(t)]$ be an exponential Riordan matrix and let $c(t)=\sum_{n=0}^{\infty} c_{n} t^{n}, r(t)=\sum_{n=0}^{\infty} r_{n} t^{n}$ be two formal power series such that $r(h(t))=h^{\prime}(t)$, and $c(h(t))=$ $g^{\prime}(t) / g(t)$. Then $a_{n+1, k}=(1 / k !) \sum_{i \geq k-1} i !\left(c_{i-k}+k r_{i-k+1}\right) a_{n, i}$, where defining $c_{-1}=0$. Conversely, starting from the sequences $c(t)=\sum_{n=0}^{\infty} c_{n} t^{n}$, and $r(t)=\sum_{n=0}^{\infty} r_{n} t^{n}$, the infinite array $\left(a_{n, k}\right)_{n, k \geq 0}$ defined by above relations is an exponential Riordan matrix.

From this proposition, the production matrix of $A=$ $[g(t), h(t)]$ is $P=\left(p_{i, j}\right)_{i, j \geq 0}$ with $p_{i, j}=(i ! / j !)\left(c_{i-j}+j r_{i-j+1}\right)$ $\left(c_{-1}=0\right)$, where the numbers $c_{n}$ and $r_{n}$ are determined by $c(t)=\sum_{n=0}^{\infty} c_{n} t^{n}=g^{\prime}(\bar{f}(t)) / g(\bar{f}(t)), r(t)=\sum_{n=0}^{\infty} r_{n} t^{n}=$ $f^{\prime}(\bar{f}(t))$. The sequence $\left(c_{n}\right)_{n \geq 0}$ and $\left(r_{n}\right)_{n \geq 0}$ are called, respectively, the $c$-sequence and the $r$-sequence of exponential Riordan array $A=[g(t), h(t)]$.

As well known, many polynomial sequences like Laguerre polynomials, first and second kind Meixner polynomials, Poisson-Charlier polynomials, and Stirling polynomials form Sheffer sequences. The Sheffer polynomials are specified here by means of the following generating function (see $[14,15]$ and the references cited therein):

$$
\sum_{n=0}^{\infty} s_{n}(x) \frac{t^{n}}{n !}=\frac{1}{g(\bar{f}(t))} e^{x \bar{f}(t)}
$$

where $f(t)$ is a delta series and $g(t)$ an invertible series, and $\bar{f}(t)$ is the compositional inverse of $f(t)$. The sequence $s_{n}(x)$ in (5) is called the Sheffer sequence for the pair $(g(t), f(t))$. The Sheffer sequence, $p_{n}(x)$ for $(1, f(t))$ is called the associated sequence and it satisfies binomial formula $p_{n}(x+y)=\sum_{k=0}^{n} p_{k}(x) p_{n-k}(y)$, so we also say that it is of binomial type. In [13], the author proved the following theorem ([13, Theorem 2.4]).

Lemma 2. Let $[g(t), f(t)]$ be an exponential Riordan array with the c-sequences $\left(c_{i}\right)_{i \geq 0}$ and $r$-sequences $\left(r_{i}\right)_{i \geq 0}$. Let $\left(a_{n}(x)\right)_{n \geq 0}$ be the Sheffer polynomial sequence for $(g(t), f(t))$. Then $a_{0}(x)=1, a_{1}(x)=x-c_{0}$, and for all $n \geq 0, a_{n+1}(x)=$ $\operatorname{det}\left(x I_{n+1}-P_{n+1}\right)$, where $P_{n+1}$ is the $(n+1)$ th principal submatrix of the production matrix $P$. 


\section{Bessel Polynomials and Bessel Matrices}

For $n \geq k \geq 0$, the signless Bessel number of the first kind $a(n, k)$ is defined to be the coefficient of $z^{n-k}$ in Bessel polynomial $y_{n-1}(z)$ with $a(0,0)=1$. The infinite lower triangular matrix $A$ with generic entry $a(n, k)$ is called signless Bessel matrix of the first kind. From [4], the signless Bessel matrix of the first kind can be represented as the exponential Riordan array $A=[1,1-\sqrt{1-2 t}]$.

Let $p_{n}(x)=x^{n} y_{n-1}(1 / x)$ for $n \geq 1$ and $p_{0}(x)=1$. Then the coefficients matrix of polynomial sequence $\left\{p_{n}(x)\right\}_{n \geq 0}$ is the signless Bessel matrix of the first kind $A=[1,1-\sqrt{1-2 t}]$. Let $\theta_{n}(x)=x^{n} y_{n}(1 / x)$ for $n \geq 0$. Then $\theta_{n}(x)=(1 / x) p_{n+1}(x)$, and $\theta_{n}(x)=x^{n} y_{n}(1 / x)=\sum_{k=0}^{n} a(n+1, k+1) x^{k}$. By [10], the infinite lower triangular matrix $B$ with generic entry $B_{n, k}=a(n+1, k+1)$ is the exponential Riordan array $B=$ $[1 / \sqrt{1-2 t}, 1-\sqrt{1-2 t}]$. We call this matrix the Bessel matrix since it is the coefficients matrix of the Bessel polynomials. From (4), one has

$$
\begin{gathered}
\sum_{n=0}^{\infty} p_{n}(x) \frac{t^{n}}{n !}=e^{x(1-\sqrt{1-2 t})}, \\
\sum_{n=0}^{\infty} \theta_{n}(x) \frac{t^{n}}{n !}=\frac{1}{\sqrt{1-2 t}} e^{x(1-\sqrt{1-2 t})} .
\end{gathered}
$$

Hence we obtain the following results (see also $[14,16]$ ).

Theorem 3. The polynomial sequence $\left\{p_{n}(x)\right\}_{n \geq 0}$ is of binomial type, and the polynomial sequence $\left\{\theta_{n}(x)\right\}_{n \geq 0}$ is a Sheffer sequence.

The generalized Bessel polynomial $y_{n}(z, a, b)$ was defined by Krall and Frink [2] as the polynomial solution of the differential equation

$$
\begin{aligned}
z^{2} y_{n}^{\prime \prime}(z)+(a z+b) y_{n}^{\prime}(z)=n(n+a-1) y_{n}(z), & \\
& b \neq 0, a \neq 0,-1,-2, \ldots .
\end{aligned}
$$

The explicit expression of the generalized Bessel polynomials is

$$
y_{n}(z, a, b)=\sum_{k=0}^{n}\left(\begin{array}{l}
n \\
k
\end{array}\right)\left(\begin{array}{c}
n+k+a-2 \\
k
\end{array}\right) k !\left(\frac{z}{b}\right)^{k} .
$$

In addition these polynomials satisfy the three-term recursion relation $(n+a-1)(2 n+a-2) y_{n+1}(z)=[(2 n+a)(2 n+$ $a-2)(z / b)+a-2](2 n+a-1) y_{n}(z)+n(2 n+a) y_{n-1}(z), n \geq 2$, with initial conditions $y_{0}(z)=1$ and $y_{1}(z)=1+a(z / b)$.

We introduce a two-variable polynomial sequence $p_{n}(z, x)$ by $p_{n}(z, x)=(2 z)^{n} p_{n}(x / 2 z)$. Then $p_{n}(z, x)=$ $\sum_{k=0}^{n} a(n, k) 2^{n-k} z^{n-k} x^{k}=\sum_{k=0}^{n}\left(\begin{array}{c}n-1 \\ k-1\end{array}\right)\left(\begin{array}{c}2 n-k-1 \\ n-1\end{array}\right)(n-k) ! z^{n-k} x^{k}$.

Theorem 4. $\left\{p_{n}(z, x)\right\}_{n \geq 0}$ is a sequence of polynomials of binomial type in the variable $x$.
Proof. From Theorem 3, we obtain

$$
\begin{aligned}
\sum_{n=0}^{\infty} p_{n}(z, x) \frac{t^{n}}{n !} & =\sum_{n=0}^{\infty}(2 z)^{n} p_{n}\left(\frac{x}{2 z}\right) \frac{t^{n}}{n !} \\
& =\sum_{n=0}^{\infty} p_{n}\left(\frac{x}{2 z}\right) \frac{(2 z t)^{n}}{n !}=e^{x((1-\sqrt{1-4 z t}) / 2 z)} .
\end{aligned}
$$

Using (5), we obtain the desired result.

From the previous proof, the polynomial sequence $p_{n}(z, x)$ has exponential generating function

$$
\sum_{n=0}^{\infty} p_{n}(z, x) \frac{t^{n}}{n !}=e^{x((1-\sqrt{1-4 z t}) / 2 z)},
$$

and $p_{n}(x)=p_{n}(1 / 2, x)$. So $\left\{p_{n}(z, x)\right\}_{n \geq 0}$ is a generalization of $\left\{p_{n}(x)\right\}_{n \geq 0}$.

Instead of working with $y_{n}(z, a, b)$, it is somewhat easier to work with the polynomials $s_{n}(z, a, x)$ defined as $s_{n}(z, a, x)=\sum_{k=0}^{n}\left(\begin{array}{c}n \\ k\end{array}\right)\left(\begin{array}{c}n+k+a-2 \\ k\end{array}\right) k ! z^{k} x^{n-k}$, which are related to the $y_{n}(z, a, b)$ by $s_{n}(z, a, x)=x^{n} y_{n}(z, a, x), n \geq 0$. It is easy to check that $s_{n}(1 / 2,2, x)=\theta_{n}(x)$. So $\left\{s_{n}(z, a, x)\right\}_{n \geq 0}$ is a generalization of $\left\{\theta_{n}(x)\right\}_{n \geq 0}$.

Theorem 5. $\left\{s_{n}(z, a, x)\right\}_{n \geq 0}$ is a Sheffer polynomial sequence in the variable $x$.

Proof. Let $B(z)=\left[(1 / \sqrt{1-4 z t})((1-\sqrt{1-4 z t}) / 2 z t)^{a-2},(1-\right.$ $\sqrt{1-4 z t}) / 2 z]$. By $(5.72)$ of [17, page 203], one has the general term of $B(z)$ which is $B(z)_{n, k}=$ $\left[t^{n} / n !\right](1 / \sqrt{1-4 z t}) t^{k}((1-\sqrt{1-4 z t}) / 2 z t)^{k+a-2}=$ $\left[t^{n} / n !\right] t^{k} \sum_{n=0}^{\infty}\left(\begin{array}{c}2 n+k+a-2 \\ n\end{array}\right)(z t)^{n}=\left(\begin{array}{c}n \\ k\end{array}\right)\left(\begin{array}{c}2 n-k+a-2 \\ n-k\end{array}\right)(n-k) ! z^{n-k}$. Hence $\quad \sum_{k=0}^{n} B(z)_{n, k} x^{k}=\sum_{k=0}^{n}\left(\begin{array}{c}n \\ k\end{array}\right)\left(\begin{array}{c}2 n-k+a-2 \\ n-k\end{array}\right)(n \quad-$ $k) ! z^{n-k} x^{k}=\sum_{k=0}^{n}\left(\begin{array}{c}n \\ n-k\end{array}\right)\left(\begin{array}{c}2 n-k+a-2 \\ n-k\end{array}\right)(n-k) ! z^{n-k} x^{k}=$ $\sum_{k=0}^{n}\left(\begin{array}{c}n \\ k\end{array}\right)\left(\begin{array}{c}n+k+a-2 \\ k\end{array}\right) k ! z^{k} x^{n-k}=x^{n} \sum_{k=0}^{n}\left(\begin{array}{c}n \\ k\end{array}\right)\left(\begin{array}{c}n+k+a-2 \\ k\end{array}\right) k !(z / x)^{k}=$ $x^{n} y_{n}(z, a, x)=s_{n}(z, a, x)$. Therefore, the exponential generating function of the sequence $\left\{s_{n}(z, a, x)\right\}_{n \geq 0}$ is

$$
\begin{aligned}
\sum_{n=0}^{\infty} s_{n}(z, a, x) \frac{t^{n}}{n !}= & \frac{1}{\sqrt{1-4 z t}}\left(\frac{1-\sqrt{1-4 z t}}{2 z t}\right)^{a-2} \\
& \times e^{x((1-\sqrt{1-4 z t}) / 2 z)} .
\end{aligned}
$$

Using (5), we obtain the desired result.

From Theorems 4 and 5, we get the following theorem.

Theorem 6. The sequences $\left\{p_{n}(z, x)\right\}_{n \geq 0}$ and $\left\{s_{n}(z, a, x)\right\}_{n \geq 0}$ satisfy the following convolution formulas:

$$
\begin{gathered}
p_{n}(z, x+y)=\sum_{k=0}^{n} p_{k}(z, x) p_{n-k}(z, y), \\
s_{n}(z, a, x+y)=\sum_{k=0}^{n} s_{k}(z, a, x) p_{n-k}(z, y) .
\end{gathered}
$$


Note that the coefficients matrix of the polynomial sequence $\left\{p_{n}(z, x)\right\}_{n \geq 0}$ is $A(z)=[1,(1-\sqrt{1-4 z t}) / 2 z]$, which is a generalization of the signless Bessel matrix of the first kind $A=[1,1-\sqrt{1-2 t}]$ while the coefficients matrix of the polynomial sequence $\left\{s_{n}(z, a, x)\right\}_{n \geq 0}$ is $B(z, a)=\left[(1 / \sqrt{1-4 z t})((1-\sqrt{1-4 z t}) / 2 z t)^{a-2},(1-\right.$ $\sqrt{1-4 z t}) / 2 z]$, which is a generalization of the Bessel matrix $B=[1 / \sqrt{1-2 t}, 1-\sqrt{1-2 t}]$.

\section{Determinant Formulae for Bessel Polynomials}

The Catalan numbers, $C_{n}=(1 /(n+1))\left(\begin{array}{c}2 n \\ n\end{array}\right)$, may be defined by the ordinary generating function $\sum_{n=0}^{\infty} C_{n} t^{n}=(1-$ $\sqrt{1-4 t}$ ) $/ 2 t$. The formulae (11) and (12) imply the connection between the generalized Bessel polynomials and Catalan numbers. In the following we will give some determinant expressions for Bessel polynomials, in which the entries involve Catalan numbers.

Define $P(z, a)$ to be the infinite Hessenberg matrix with general entry

$$
p_{i, j}(z, a)= \begin{cases}1, & \text { if } i=j-1, \\ -\frac{i !}{j !}(2 i+a) C_{i-j} z^{i-j+1}, & \text { if } i \geq j \geq 0, \\ 0, & \text { otherwise. }\end{cases}
$$

The first rows of $P$ are

$$
P(z, a)=\left(\begin{array}{ccccc}
-a C_{0} z & 1 & 0 & 0 & \cdots \\
-(2+a) C_{1} z^{2} & -(2+a) C_{0} z & 1 & 0 & \cdots \\
-2(4+a) C_{2} z^{3} & -2(4+a) C_{1} z^{2} & -(4+a) C_{0} z & 1 & \cdots \\
-6(6+a) C_{3} z^{4} & -6(6+a) C_{2} z^{3} & -3(6+a) C_{1} z^{2} & -(6+a) C_{0} z & \cdots \\
-24(8+a) C_{4} z^{5} & -24(8+a) C_{3} z^{4} & -12(8+a) C_{2} z^{3} & -4(8+a) C_{1} z^{2} & \cdots \\
\vdots & \vdots & \vdots & \vdots & \ddots
\end{array}\right)
$$

Theorem 7. For $n=0,1,2, \ldots$, the polynomial $s_{n+1}(z$, $a, x)$ admits the following determinant representa- tion $s_{n+1}(z, a, x)=\operatorname{det}\left(x I_{n+1}-P_{n+1}(z, a)\right)$, that is,

$$
s_{n+1}(z, a, x)=\left|\begin{array}{ccccc}
x+a C_{0} z & -1 & 0 & \cdots & 0 \\
(2+a) C_{1} z^{2} & x+(2+a) C_{0} z & -1 & \cdots & 0 \\
2(4+a) C_{2} z^{3} & 2(4+a) C_{1} z^{2} & x+(4+a) C_{0} z & \cdots & 0 \\
\vdots & \vdots & \vdots & \ddots & \vdots \\
\frac{n !(2 n+a)}{0 !} C_{n} z^{n+1} & \frac{n !(2 n+a)}{1 !} C_{n-1} z^{n} & \frac{n !(2 n+a)}{2 !} C_{n-2} z^{n-1} & \cdots & x+(2 n+a) C_{0} z
\end{array}\right|
$$

where $P_{n+1}(z, a)$ is the $(n+1)$ th principal submatrix of Hessenberg matrix $P(z, a)$ defined by (14).

Proof. Let $g(t)=(1-2 z t)(1-z t)^{a-2}$ and $f(t)=t(1-z t)$; then $\bar{f}(t)=(1-\sqrt{1-4 z t}) / 2 z, 1 / g(\bar{f}(t))=(1 / \sqrt{1-4 z t})((1-$ $\sqrt{1-4 z t}) / 2 z t)^{a-2}$. Therefore, from (12), $\left\{s_{n}(z, a, x)\right\}_{n \geq 0}$ is a Sheffer polynomial sequence for the pair $((1-2 z t)(1-$ $\left.z t)^{a-2}, t(1-z t)\right)$.
The generating functions for the $r$-sequence and $c$ sequence of $[g(t), f(t)]$ are $r(t)=f^{\prime}(\bar{f}(t))=\sqrt{1-4 z t}=1-$ $2 \sum_{n=1}^{\infty} C_{n-1} z^{n} t^{n}, c(t)=g^{\prime}(\bar{f}(t)) / g(\bar{f}(t))=(2-a) z C(z t)-$ $2 z /(\sqrt{1-4 z t})=\sum_{n=0}^{\infty}-(2 n+a) C_{n} z^{n+1} t^{n}$. By Lemma 1 , the production matrix of $[g(t), f(t)]$ is exactly the Hessenberg matrix $P$ defined by (14). Hence, by Lemma 2, we obtain the desired result.

Corollary 8. For $n=0,1,2, \ldots$, one has $y_{n+1}(z, a, x)=$ $\operatorname{det}\left(I_{n+1}-(1 / x) P_{n+1}(z, a)\right)$. Particularly, 


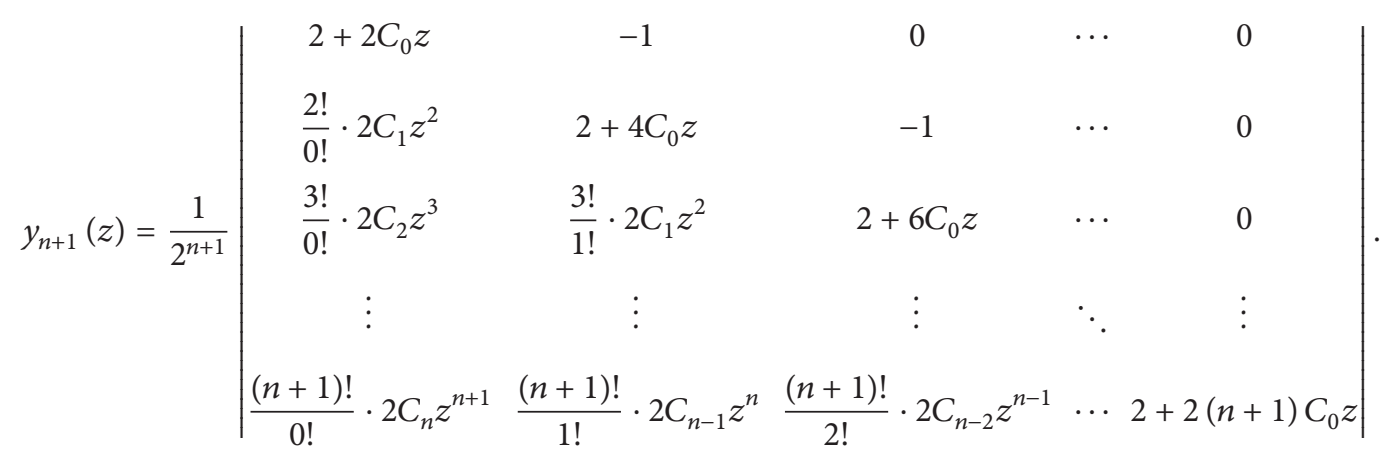

Corollary 9. For $n=0,1,2, \ldots$, we have

$$
\begin{aligned}
& \theta_{n+1}(x)=\left|\begin{array}{ccccc}
x+C_{0} & -1 & 0 & \cdots & 0 \\
\frac{2 !}{0 ! 2} C_{1} & x+2 C_{0} & -1 & \cdots & 0 \\
\frac{3 !}{0 ! 2^{2}} C_{2} & \frac{3 !}{1 ! 2} C_{1} & x+3 C_{0} & \cdots & 0 \\
\vdots & \vdots & \vdots & \ddots & \vdots \\
\frac{(n+1) !}{0 ! 2^{n}} C_{n} & \frac{(n+1) !}{1 ! 2^{n-1}} C_{n-1} & \frac{(n+1) !}{2 ! 2^{n-2}} C_{n-2} & \cdots & x+(n+1) C_{0}
\end{array}\right|
\end{aligned}
$$

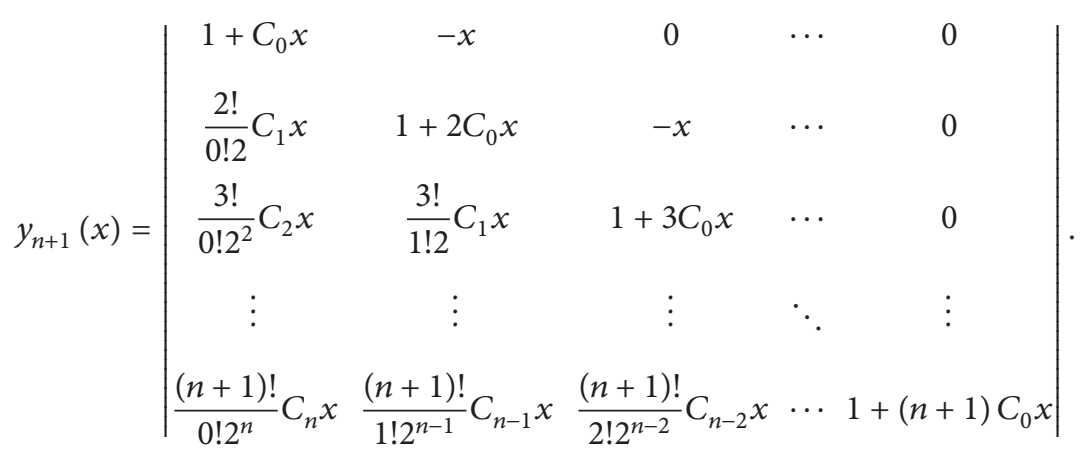

Examples. (1) One has

$$
\begin{aligned}
& s_{3}(z, a, x) \\
& =\left|\begin{array}{ccc}
x+a C_{0} z & -1 & 0 \\
(2+a) C_{1} z^{2} & x+(2+a) C_{0} z & -1 \\
2(4+a) C_{2} z^{3} & 2(4+a) C_{1} z^{2} & x+(4+a) C_{0} z
\end{array}\right| \\
& =x^{3}+3(a+2) z x^{2}+3(a+2)(a+3) z^{2} x \\
& +(a+2)(a+3)(a+4) z^{3} \text {. } \\
& y_{3}(z, a, x) \\
& =\left(\frac{1}{x^{3}}\right)\left|\begin{array}{ccc}
x+a C_{0} z & -1 & 0 \\
(2+a) C_{1} z^{2} & x+(2+a) C_{0} z & -1 \\
2(4+a) C_{2} z^{3} & 2(4+a) C_{1} z^{2} & x+(4+a) C_{0} z
\end{array}\right| \\
& =1+3(a+2)\left(\frac{z}{x}\right)+3(a+2)(a+3)\left(\frac{z}{x}\right)^{2} \\
& +(a+2)(a+3)(a+4)\left(\frac{z}{x}\right)^{3} \text {. }
\end{aligned}
$$


(3) Consider the following:

$$
\begin{aligned}
y_{3}(z) & =\left(\frac{1}{2^{3}}\right)\left|\begin{array}{ccc}
2+2 z & -1 & 0 \\
4 z^{2} & 2+4 z & -1 \\
24 z^{3} & 12 z^{2} & 2+6 z
\end{array}\right| \\
& =1+6 z+15 z^{2}+15 z^{3} .
\end{aligned}
$$

(4) Consider the following:

$$
\begin{aligned}
\theta_{3}(x) & =\left|\begin{array}{ccc}
x+1 & -1 & 0 \\
1 & x+2 & -1 \\
3 & 3 & x+3
\end{array}\right| \\
& =x^{3}+6 x^{2}+15 x+15 .
\end{aligned}
$$

(5) One has

$$
\begin{aligned}
y_{3}(x) & =\left|\begin{array}{ccc}
1+x & -x & 0 \\
x & 1+2 x & -x \\
3 x & 3 x & 1+3 x
\end{array}\right| \\
& =15 x^{3}+15 x^{2}+6 x+1 .
\end{aligned}
$$

\section{Acknowledgments}

This work was supported by the National Natural Science Foundation of China (Grant no. 11261032) and the Natural Science Foundation of Gansu Province (Grant no. 1010RJZA049).

\section{References}

[1] E. Grosswald, Bessel Polynomials, Springer, New York, NY, USA, 1978.

[2] H. L. Krall and O. Frink, "A new class of orthogonal polynomials: the Bessel polynomials," Transactions of the American Mathematical Society, vol. 65, pp. 100-115, 1949.

[3] H. Han and S. Seo, "Combinatorial proofs of inverse relations and log-concavity for Bessel numbers," European Journal of Combinatorics, vol. 29, no. 7, pp. 1544-1554, 2008.

[4] S. L. Yang and Z. K. Qiao, "The Bessel numbers and Bessel matrices," Journal of Mathematical Research and Exposition, vol. 31, no. 4, pp. 627-636, 2011.

[5] T. Mansour, M. Schork, and M. Shattuck, "The generalized Stirling and Bell numbers revisited," Journal of Integer Sequences, vol. 15, no. 8, article 12.8.3, 2012.

[6] S. K. Chatterjea, "On the Bessel polynomials," The Mathematical Journal of the University of Padova, vol. 32, pp. 295-303, 1962.

[7] Ö. Eğecioğlu, "Bessel polynomials and the partial sums of the exponential series," SIAM Journal on Discrete Mathematics, vol. 24, no. 4, pp. 1753-1762, 2010.

[8] L. W. Shapiro, S. Getu, W. J. Woan, and L. C. Woodson, "The Riordan group," Discrete Applied Mathematics, vol. 34, no. 1-3, pp. 229-239, 1991.

[9] P. Barry, "On a family of generalized Pascal triangles defined by exponential Riordan arrays," Journal of Integer Sequences, vol. 10, no. 3, article 07.3.5, 2007.

[10] E. Deutsch, L. Ferrari, and S. Rinaldi, "Production matrices and Riordan arrays," Annals of Combinatorics, vol. 13, no. 1, pp. 6585, 2009.
[11] T.-X. He and R. Sprugnoli, "Sequence characterization of Riordan arrays," Discrete Mathematics, vol. 309, no. 12, pp. 39623974, 2009.

[12] P. Peart and W.-J. Woan, "Generating functions via Hankel and Stieltjes matrices," Journal of Integer Sequences, vol. 3, no. 2, article 00.2.1, 2000.

[13] S.-l. Yang, "Recurrence relations for the Sheffer sequences," Linear Algebra and Its Applications, vol. 437, no. 12, pp. 29862996, 2012.

[14] S. Khan, M. W. Al-Saad, and G. Yasmin, "Some properties of Hermite-based Sheffer polynomials," Applied Mathematics and Computation, vol. 217, no. 5, pp. 2169-2183, 2010.

[15] S. Roman, The Umbral Calculus, vol. 111, Academic Press, New York, NY, USA, 1984.

[16] L. Carlitz, "A note on the Bessel polynomials," Duke Mathematical Journal, vol. 24, pp. 151-162, 1957.

[17] R. L. Graham, D. E. Knuth, and O. Patashnik, Concrete Mathematics, Addison-Wesley, New York, NY, USA, 2nd edition, 1994. 


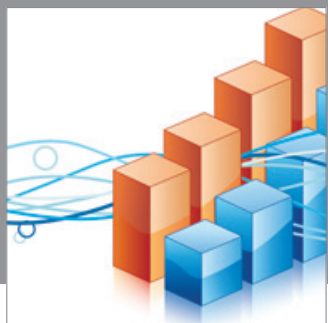

Advances in

Operations Research

mansans

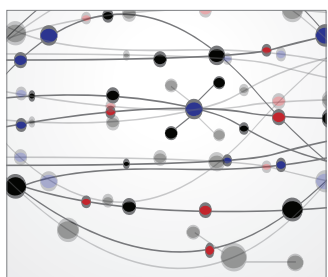

The Scientific World Journal
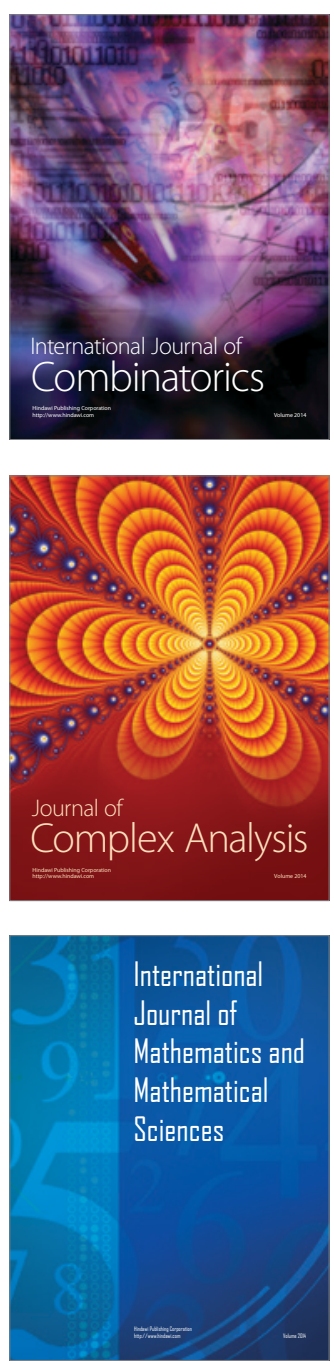
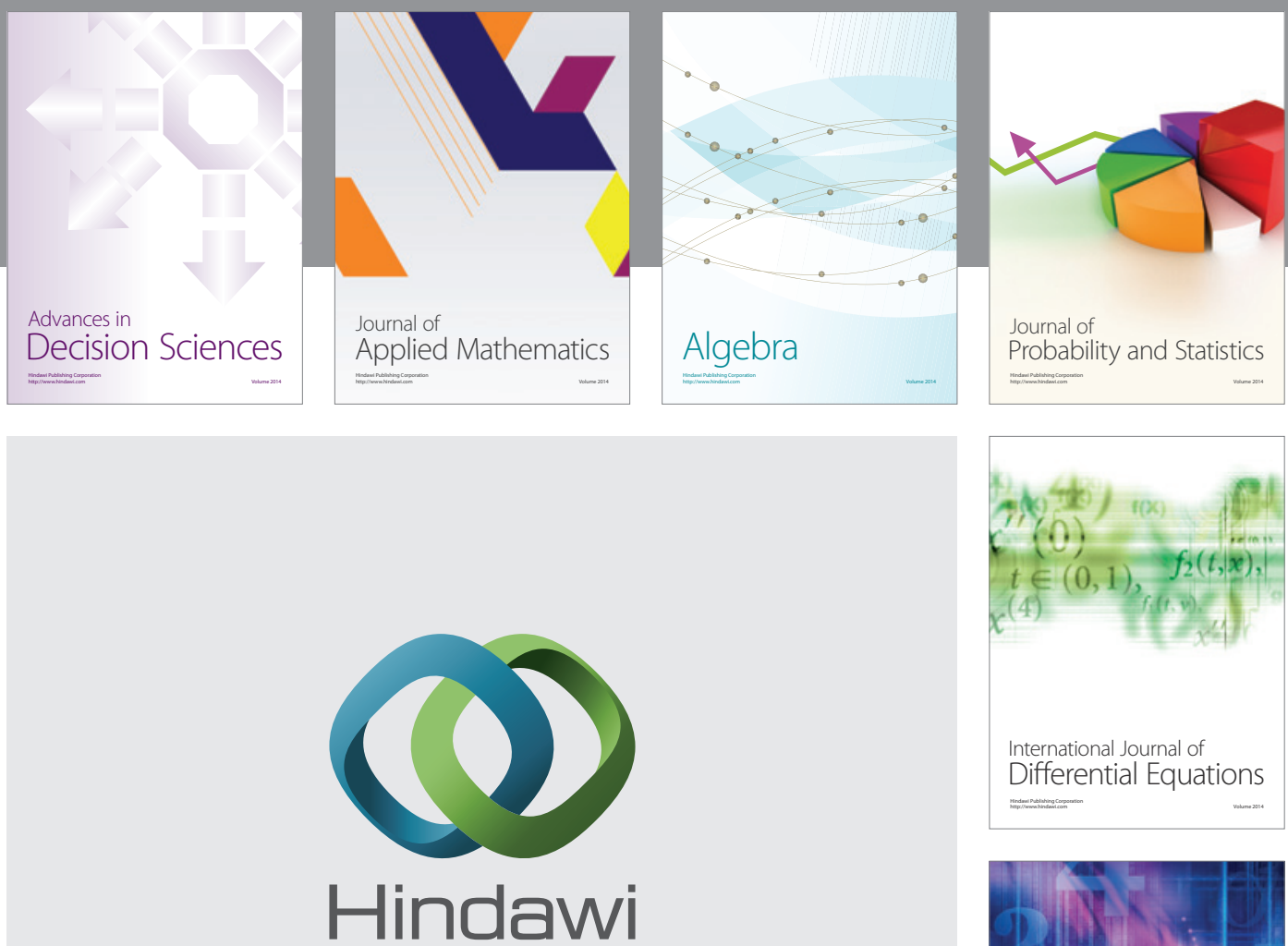

Submit your manuscripts at http://www.hindawi.com
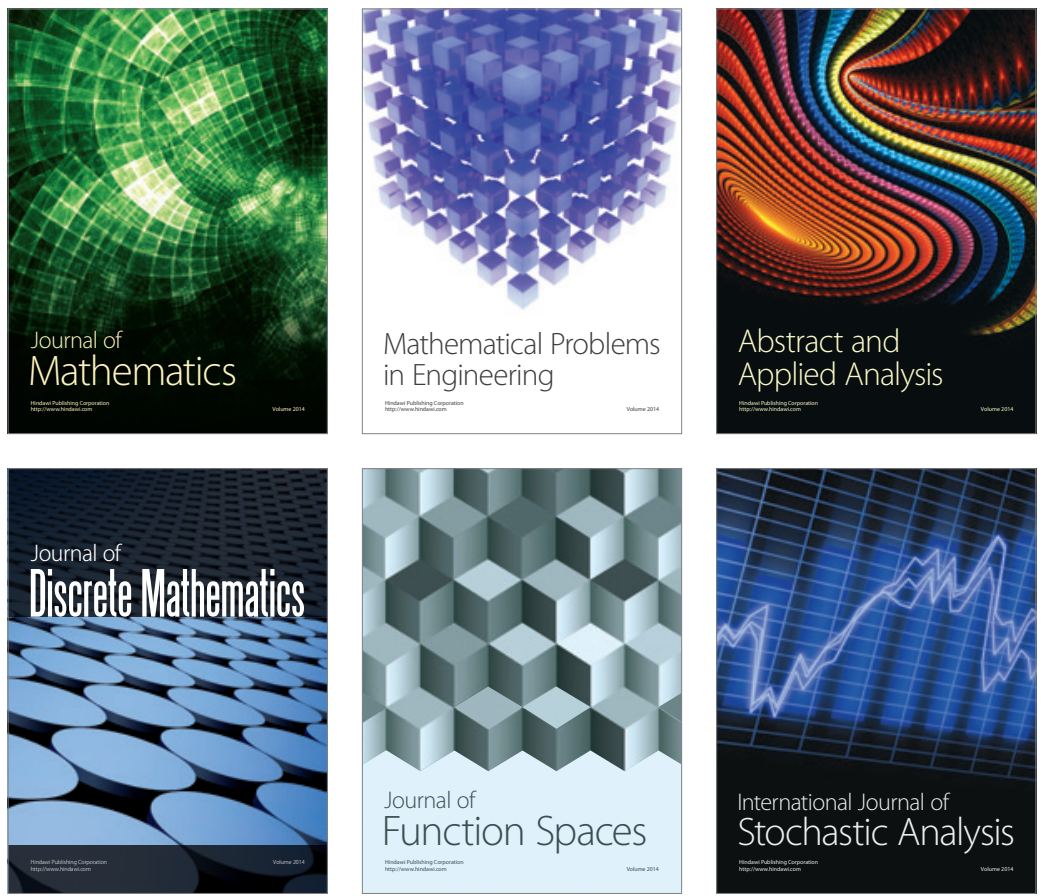

Journal of

Function Spaces

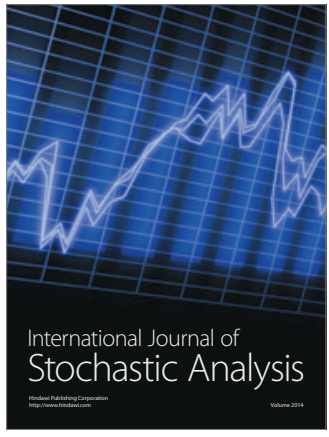

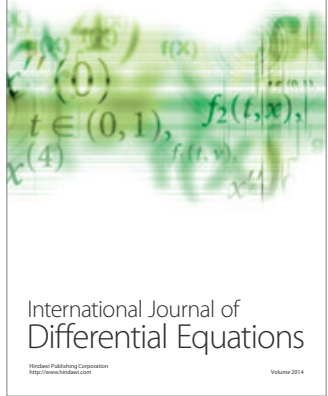
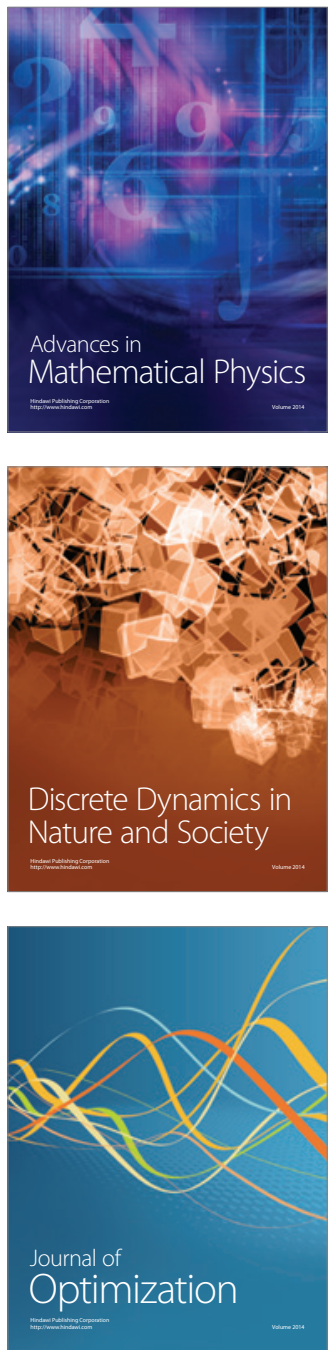